\title{
Task-shifting point-of-care CD4+ testing to lay health workers in HIV care and treatment services in Namibia
}

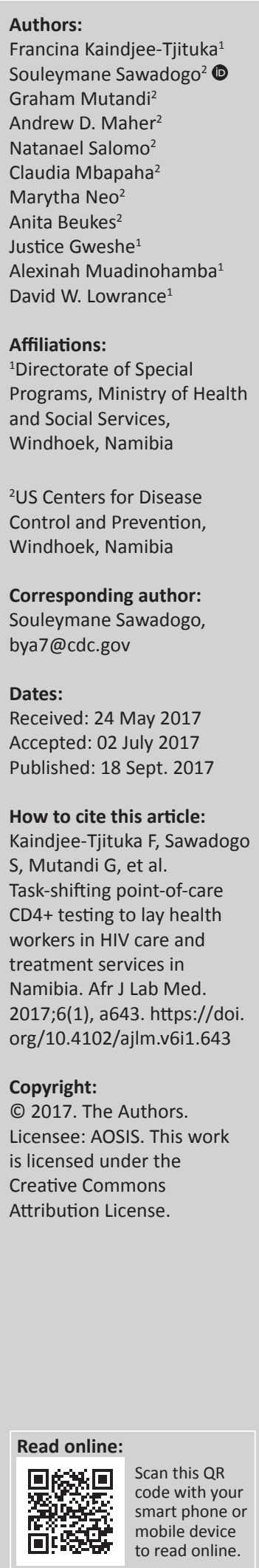

Introduction: Access to CD4+ testing remains a common barrier to early initiation of antiretroviral therapy among persons living with HIV/AIDS in low- and middle-income countries. The feasibility of task-shifting of point-of-care (POC) CD4+ testing to lay health workers in Namibia has not been evaluated.

Methods: From July to August 2011, Pima CD4+ analysers were used to improve access to CD4+ testing at 10 selected public health facilities in Namibia. POC Pima CD4+ testing was performed by nurses or lay health workers. Venous blood samples were collected from $10 \%$ of patients and sent to centralised laboratories for CD4+ testing with standard methods. Outcomes for POC Pima CD4+ testing and patient receipt of results were compared between nurses and lay health workers and between the POC method and standard laboratory CD4+ testing methods.

Results: Overall, 1429 patients received a Pima CD4+ test; 500 (35.0\%) tests were performed by nurses and 929 (65.0\%) were performed by lay health workers. When Pima CD4+ testing was performed by a nurse or a lay health worker, $93.2 \%$ and $95.2 \%$ of results were valid $(p=0.1)$; $95.6 \%$ and $98.1 \%$ of results were received by the patient ( $p=0.007) ; 96.2 \%$ and $94.0 \%$ of results were received by the patient on the same day $(p=0.08)$. Overall, $97.2 \%$ of Pima CD $4+$ results were received by patients, compared to $55.4 \%$ of standard laboratory CD $4+$ results $(p<0.001)$.

Conclusions: POC CD4+ testing was feasible and effective when task-shifted to lay health workers. Rollout of POC CD4+ testing via task-shifting can improve access to CD4+ testing and retention in care between HIV diagnosis and antiretroviral therapy initiation in low- and middle-income countries.

\section{Introduction}

In 2012, United Nations Programme on HIV / AIDS (UNAIDS) reported that only about 8 million of the 15 million people in low- and middle-income countries (LMIC) in need of antiretroviral therapy (ART) were receiving ART by the end of 2011. ${ }^{1}$ In the LMIC of sub-Saharan Africa, many barriers exist to early initiation of ART among eligible HIV-positive patients. Of these barriers, limited access to CD4+ testing for determination of ART eligibility is one of the most common. ${ }^{2,3,4,5,6,7}$ A recent systematic review of 28 published studies examining the retention of patients in sub-Saharan Africa in HIV care between testing and treatment found that the median proportion of patients retained between HIV diagnosis and receipt of CD4+ test results was 59\% (range: $35 \%-88 \%)^{8}$

Implementation of rapid, point-of-care (POC) CD4+ testing in HIV care and treatment (HCT) settings has been shown to increase the number of patients who undergo and receive the results of CD4+ testing, and has the potential to improve retention between HIV diagnosis and ART initiation. ${ }^{9}$ The Pima ${ }^{\circledR}$ POC CD4+ analyser (Alere) has been validated in the field against standardof-care, laboratory-based platforms $\mathrm{s}^{10,11,12,13}$ and was listed as a World Health Organization (WHO) prequalified diagnostic in November 2011. ${ }^{14}$ Recent field-based studies in Mozambique and South Africa demonstrated that POC testing with the Pima CD4+ enabled clinics to rapidly stage patients on-site after enrolment into care, which reduced opportunities for pre-treatment loss to follow-up. ${ }^{15,16}$ POC CD4 testing has also been successfully deployed in mobile, community-based and household settings in South Africa. ${ }^{17,18}$

Task-shifting to meet human resources for health needs is recommended by WHO. ${ }^{19}$ Because POC CD4+ testing using the Pima CD4+ analyser does not require a laboratory and is relatively easy to perform, the task of performing CD4+ tests in the HCT setting could potentially be shifted from nurses and laboratory technicians to non-professional or lay health workers. 
In Namibia, lay health workers in the 'community counsellors' cadre have been trained and certified by the Namibia Ministry of Health and Social Services (MOHSS) to provide health facility-based HIV testing and counselling. Roll-out and scale-up of POC CD4+ testing in HCT settings could follow a similar model. We assessed the feasibility of POC CD4+ testing performed by nurses or lay health workers and the effectiveness of POC CD4+ testing in reducing result turn-around time (TAT) and increasing the proportion of patients who receive their $\mathrm{CD} 4+$ test results.

\section{Methods}

\section{Ethical considerations}

Prior to implementation, the study protocol was approved by the Research Committee of the Namibia Institute of Pathology and the US Centers for Disease Control and Prevention. The analysis of the data collected for this study was approved as 'non-research' by the US Centers for Disease Control and Prevention and the Office of the Permanent Secretary of the MOHSS of Namibia. Informed patient consent is not required for receipt of routine services (including CD4 count testing) performed at MOHSS facilities and no personally identifiable information was available to the researchers; thus, written informed consent was not collected from patients.

\section{Study design}

In July 2011, the Namibia MOHSS began a multi-phased rollout of POC CD4+ testing using the Pima CD4+ analyser in select public sector HCT clinics throughout the country. Prior to the first phase of the rollout of POC CD4+ testing, nurses and lay health workers received theoretical and practical training in use of the Pima analyser. Trainees were required to pass a written examination designed by the device manufacturer (Alere, Waltham, Massachusetts, United States) prior to performing testing in this study. From July 2011 to August 2011, Pima CD4+ POC tests were used for routine CD4+ testing for both baseline assessment of ART eligibility and routine ART monitoring among adult patients (age $\geq 15$ years) receiving HCT services at 10 public clinics scattered across Namibia selected purposively by virtue of having an on-site laboratory. Lay health worker and nurse testers were also purposively selected for testing during this study by virtue of availability at these 10 clinics.

\section{Testing procedures}

All patients eligible to receive standard-of-care CD4 testing at the participating clinics during the study period were consecutively sampled. Pima CD4+ testing was performed by trained nurses or lay health workers and both cadres were represented at all 10 sites. Patient management actions including initiation of ART, referral for pre-ART follow-up and requisition of further laboratory tests were initiated by clinicians based on the Pima CD4+ result of each patient. For the purpose of comparing some CD4+ results, TAT and patient receipt of results for Pima CD4+ and standard testing methods, venous blood was collected by nurses from approximately $10 \%$ of participants and sent to the laboratory for standard CD4+ testing on either the EPICS XL or FC500 (Beckman-Coulter, Brea, California, United States); results were returned to the clinic upon availability.

\section{Data collection}

The following data were recorded by nurses or lay health workers in study-specific registers: location, date and time of specimen collection, testing cadre, validity (i.e. 'valid' or 'invalid') of each Pima CD4+ test result, time of Pima CD4+ test result availability, time of test result disclosure to the patient, Pima CD4+ result and patient demographic information, including age, sex and reason for CD4+ testing (i.e. ART eligibility assessment or ART monitoring). A questionnaire was sent to all nurses and lay health workers by the study staff to rate their experience with using the Pima CD4+ test towards the end of the study period. The rating categories ranged from very easy, easy, fair, difficult to very difficult. The times of specimen collection and patient receipt of results for standard laboratory CD4+ testing were also collected. Patient management actions initiated by clinicians following receipt of POC CD4+ results were recorded.

\section{Analysis}

Basic patient demographic and clinical characteristics were calculated and stratified by sex and reason for CD4+ testing. The feasibility of task-shifting POC CD4+ testing to lay health workers was assessed by calculating the following for both nurses and lay health workers: percentage of valid Pima CD4+ test results produced, percentage of Pima CD4+ test results received by patients overall and received by patients on the same day as testing, median Pima CD4+ result TAT and percentage of eligible patients (i.e. Pima CD4+ result $\leq 350$ cells $/ \mu \mathrm{L}$ ) who initiated ART at the same or next clinic visit.

All percentages were calculated at the 95\% confidence level. Two-proportion z-tests and Pearson's chi-square or the Mann-Whitney test (for median result TAT only), were used to test for significant differences between the tester cadres. The effectiveness of POC CD4+ testing was assessed by calculating the percentage of patients who received their Pima CD4+ or standard laboratory CD4+ results and the median and interquartile range (IQR) for the TAT for both CD4+ testing methods. Two-proportion z-tests and Pearson's chi-square or the Wilcoxon matched-pairs test (for median TAT results), were used to test for significant differences between the Pima CD4+ testing and standard laboratory testing methods. The Wilcoxon test, which is non-parametric, was used due to the asymmetrical distribution of CD4+ TAT results.

\section{Results}

Overall, 1429 patients (68\% women) received a Pima CD4+ test during the study period (Table 1). Of these, 407 patients (28.5\%) received Pima CD4+ tests for an ART eligibility 
TABLE 1: Demographic and clinical characteristics of patients who received point-of-care Pima CD4+ tests performed by nurses or lay health workers, Namibia, July-August 2011.

\begin{tabular}{lcc}
\hline Variable & \multicolumn{2}{c}{ Reason for CD4+ testing } \\
\cline { 2 - 3 } Sex: $n$ (\%) & $253(26.0)$ & $719(74.0)$ \\
Women & $154(34.0)$ & $303(64.0)$ \\
Men & & $36(29-42)$ \\
Age: Median years (IQR) & $34(28-40)$ & $34(28-41)$ \\
All & $31(26-38)$ & $38(32-45)$ \\
Women & $37(32-41)$ & \\
Men & & $338(221-462)$ \\
Pima CD4+ result: Median & & \\
cells $\mu$ L (IQR), range & $222(111-363), 18-641$ & $306(182-439), 11-627$ \\
All & $233(128-365)$ & \\
Women & $202(82-363)$ & \\
Men & & \\
Eligible to initiate ART by & & \\
Pima result, i.e. CD4+ $\leq 350$ & & \\
cells $\mu$ L: $n$ (\%) & & \\
All & $283(69.5)$ & \\
Women & $107(37.8)$ & \\
Men & $176(62.2)$ & \\
\hline
\end{tabular}

ART, antiretroviral therapy; IQR, interquartile range.

assessment and 1022 patients (71.5\%) received Pima CD4+ tests for ART monitoring. Pima results ranged from 11-641 cells $/ \mu \mathrm{L}$ overall. The median Pima CD4+ result among patients tested for an ART eligibility assessment was 233 cells / $\mu$ L (IQR: 128-365) among women and 202 cells $/ \mu \mathrm{L}$ (IQR: 82-363) among men. Based on their Pima CD4+ results, $69.5 \%$ of patients were eligible to initiate ART (i.e. $\leq 350$ cells). The median Pima CD4+ result among patients tested for ART monitoring was 338 cells/ $\mu \mathrm{L}$ (IQR: 221-462) among women and 218 cells / $\mu \mathrm{L}$ (IQR: 142-358) among men.

Of the 1429 Pima CD4+ tests performed, 500 (35.0\%) were performed by nurses and $929(65.0 \%)$ by lay health workers (Table 2). Sixty-one per cent $(61 \%)$ of testers were lay health workers. Results for nurses and lay health workers were similar for the validity of Pima CD4+ test results and for receipt of results by patients on the same day as testing. A statistically significant difference between cadres was found for overall receipt of Pima CD4+ results by the patient $(95.6 \%$ for nurses, $98.1 \%$ for lay health workers; $p=0.007)$. The median result TAT was $20 \mathrm{~min}$ when performed by nurses and 21 min when performed by lay health workers $(p=0.39$ ). Among the 283 patients who were eligible to initiate ART based on the results of their Pima CD4+ test, data on patient management action taken either at the same visit when Pima CD4+ testing was performed or at the next follow-up visit were available for 226 (79.9\%). Among these, 7.7\% who were tested by nurses and $5.6 \%$ who were tested by lay health workers $(p=0.5)$ were documented as initiating ART at the same eligibility assessment visit or at the next follow-up visit. The majority of both nurses and lay health workers rated the Pima CD4+ test favourably for ease of use.

Among patients who received a Pima CD4+ test, 1389 (97.2\%) patients received their test results, of whom $1317(92.2 \%)$ received their results on the same day (Table 3). Of the 128 specimens collected for CD4+ testing by standard laboratory
TABLE 2: Comparison of outcomes for point-of-care Pima CD4+ testing performed by nurses or by lay health workers, Namibia, July-August 2011.

\begin{tabular}{lccc}
\hline Variable & \multicolumn{2}{c}{ Testing cadre } & $p^{\gamma}$ \\
\cline { 2 - 3 } & Nurses & Lay health workers & \\
\hline Pima CD4+ performed: $n$ (\%) & $500(35.0)$ & $929(65.0)$ & --- \\
$\begin{array}{l}\text { Valid Pima CD4+ performed: } \\
\text { \% (CI) }\end{array}$ & $93.2(91.0-95.0)$ & $95.2(94.0-96.6)$ & 0.1 \\
$\begin{array}{l}\text { Pima CD4+ results received by } \\
\text { patient: \% (CI) }\end{array}$ & $95.6(93.8-97.4)$ & $98.1(97.2-99.0)$ & 0.007 \\
$\begin{array}{l}\text { Pima CD4+ results received by } \\
\text { patient same day: \% (CI) }\end{array}$ & $96.2(94.5-97.9)$ & $94.0(92.5-95.6)$ & 0.08 \\
$\begin{array}{l}\text { Pima CD4+ results turnaround } \\
\text { time: median minutes (IQR) }\end{array}$ & $20(18-27)$ & $21(18-26)$ & 0.39 \\
$\begin{array}{l}\text { Eligible patient (i.e. Pima CD4 } \\
\text { 350) initiated ART }\end{array}$ & $7.7(1.1-14.2)$ & $5.6(2.0-9.2)$ & 0.5 \\
$\begin{array}{l}\text { Tester rated Pima CD4+ } \\
\text { favourably: \% (CI) }\end{array}$ & $96.6(94.0-97.5)$ & $94.7(92.9-95.9)$ & 0.4 \\
\hline
\end{tabular}
favourably: \% (CI)

ART, antiretroviral therapy; IQR, interquartile range.

$\dagger$ Significance tested by two-proportion z-test and Pearson's chi-square or Mann-Whitney test (for median result TAT only).

$\star$ All confidence intervals ( $\mathrm{Cl}$ ) are at the $95 \%$ level.

$\S$ Percentage calculated only among patients who received Pima result $(n=1389)$.

- Percentage calculated only among patients eligible to initiate ART who received results and had data on subsequent patient management action available $(n=226)$.

TABLE 3: Comparison of outcomes for point-of-care Pima CD4+ testing with outcomes for standard laboratory CD4+ testing method, Namibia, July-August 2011.

\begin{tabular}{lccc}
\hline Variable & \multicolumn{2}{c}{ Method of CD4+ test } & \multirow{2}{*}{$\boldsymbol{p}^{+}$} \\
\cline { 2 - 3 } & \multicolumn{1}{c}{ Pima } & Standard laboratory & \\
\hline $\begin{array}{l}\text { Patient received CD4+ result: } \\
\%(\mathrm{Cl})^{*, 8}\end{array}$ & $97.2(96.3-98.1)$ & $55.4(46.7-64.1)$ & $<0.001$ \\
$\begin{array}{l}\text { Patient received CD4+ result } \\
\text { same day: \%(Cl) }\end{array}$ & $92.2(90.8-93.6)$ & $4.7(0.9-8.3)$ & $<0.001$ \\
$\begin{array}{l}\text { Results turnaround time: } \\
\text { median (IQR) }\end{array}$ & 20 min (18-27) & 4 days (2-8) & \\
\hline
\end{tabular}

$\dagger$ Significance tested by two-proportion z-test and Pearson's chi-square test. $\$$ All confidence intervals (CI) are at the $95 \%$ level.

$\S$ Pima, $n=1429$; Standard laboratory, $n=128$.

methods, $71(55.4 \%)$ of results had been received by patients by the end of the study period. The median TAT for Pima CD4+ test results was $20 \mathrm{~min}$ (IQR: 18-27), whereas the median TAT for standard laboratory CD4+ test results was 4 days (IQR: 2-8).

\section{Discussion}

This study demonstrated that POC CD4+ testing was feasible and effective when performed by lay health workers. Whether performed by nurses or lay health workers, POC CD4+ testing reduced TATs for laboratory results by a median of 4 days compared to TATs for standard laboratory methods and greatly increased receipt of CD4+ test results by patients. With more than $95 \%$ of patients receiving same-day CD4+ results, the potential for attrition between HIV diagnosis and ART initiation was greatly reduced. When compared to the $59 \%$ median for the percentage of patients who were retained between HIV diagnosis and receipt of CD4+ test results in Rosen and Fox's systematic review, ${ }^{8}$ our results indicate that retention between HIV diagnosis and receipt of CD4+ results could be greatly improved by task-shifting POC CD4+ testing to nurses and lay health workers. Almost all nurses and lay health workers in our pilot rated the POC Pima CD4+ favourably on ease-of-use questionnaires; thus, this pilot provides additional evidence supporting the feasibility of task-shifting POC CD4+ testing to nurses and lay health workers in this environment. 
Extensive evidence supports such task-shifting in HCT settings in LMIC in sub-Saharan Africa. ${ }^{20,21,22,23,24,25,26,27}$ In several countries in sub-Saharan Africa that are affected by a generalised HIV epidemic and a critical shortage of skilled health care providers, scale-up of voluntary HIV testing and counselling has been facilitated by the utilisation of nonprofessional or lay health worker cadres. ${ }^{28,29}$ Results from the current study show that additional efficiencies can be gained by task-shifting POC CD4+ testing to lay health workers. Although current WHO recommendations for expanding access to POC CD4+ testing only recommend that POC diagnostics (with the exception of HIV rapid tests) be performed by first-tier clinical staff, our results support the idea that task-shifting of POC CD4+ testing and other diagnostics to lay health workers is appropriate.

In our study, POC CD4+ testing greatly increased the percentage of patients who received their CD4+ test results. However, among patients eligible to initiate ART based on their Pima CD4+ results, fewer than 10\% actually initiated ART at the baseline study visit or the next follow-up visit. Among these, $7.7 \%$ who received Pima CD4+ results from tests performed by nurses and $5.6 \%(p=0.5)$ who received results from lay health workers initiated ART at the same eligibility assessment visit or at the next follow-up visit. The possible reasons for the slightly higher percentage who initiated ART soon after a PIMA CD4+ test done by a nurse rather than a lay health worker are not clear. However, this finding may indicate that nurses who conduct the test and review the results are more likely to initiate the patient on ART themselves, thereby eliminating the potential barrier of referral to a doctor for ART initiation. In contrast, lay health workers are not qualified to interpret CD4 results, nor can they initiate patients on ART. They can only refer patients for review of results and clinical management, steps which create a potential bottleneck for ART initiation.

At the time this study was conducted in 2011, the Namibia guidelines ${ }^{30}$ recommended routine baseline tests for everyone prior to initiation of ART. This has since slightly changed with the 2014 national guidelines ${ }^{31}$ which adopted the WHO 2013 guideline recommendation ${ }^{32}$ of same-day ART initiation for some eligible patients. While still recommending baseline tests for all patients, the Namibia guidelines now recommend that all HIV-positive pregnant and breastfeeding women should be initiated on ART on the same day that ART eligibility is established, even in the absence of baseline results. This indicates that, apart from pregnant and breastfeeding women for whom baseline tests are now not mandatory to initiate ART, POC CD4+ testing alone may not remove all barriers to early initiation of ART and opportunities for pre-treatment loss to follow-up may persist without additional steps. Similarly, low rates of early ART initiation following receipt of Pima CD4+ results were observed by Jani et al. ${ }^{15}$ in their studies of Pima CD4+ in Mozambique. One contributing factor may be that additional diagnostics for the purpose of determining appropriate first-line regimens, including serum creatinine testing for contraindication to tenofovir-containing regimens $^{33,34}$ and alanine transaminase or hepatitis $B$ virus serology for contraindication to nevirapine-containing regimens, are recommended, and may be performed over several visits before ART is initiated. In Namibia, and in most LMIC, these tests are typically performed through standard laboratory-based methods in centrally located laboratories away from the POC so that, like standard laboratory CD4+ testing, patients must return at a later date to receive their results. POC diagnostics for hepatitis B virus, ${ }^{35}$ alanine transaminase, $^{36,37}$ serum creatinine ${ }^{38}$ and other tests are available and should be evaluated alongside POC CD4+ testing to further reduce opportunities for pre-treatment loss resulting from sub-optimal access to the diagnostics used to determine ART eligibility and appropriate first-line regimens.

\section{Limitations}

Several factors may limit the external validity of results from this study. Selection bias may have been introduced by purposive selection of clinics that have an on-site laboratory. Clinics with a robust infrastructure may have had better trained or more skilled staff than clinics with weaker infrastructure. Selection bias may also have been introduced by purposive selection of testers. Lay health workers and nurses were selected by virtue of their availability at the selected clinics during the study period. The lay health worker to nurse ratio in public clinics across Namibia may not reflect the lay health worker to nurse ratio in our study.

Finally, we did not assess the accuracy of Pima CD4+ results in relation to the standard laboratory-based platforms, nor did we compare accuracy of testing between lay health workers and nurses. Although the accuracy of CD4+ testing is paramount for determining eligibility and effective monitoring, the investigators felt that the accuracy of the Pima analyser had been sufficiently validated in previously published studies. Therefore, assessing the accuracy of Pima CD4+ testing was not included among the objectives or measures of this study.

\section{Conclusion}

In this study, we demonstrated that POC CD4+ testing was feasible and effective when performed by lay health workers and nurses within an HCT service delivery setting, leading to high levels of patient receipt of results and greatly improved TATs for CD4+ results. By removing one of the major sources of pre-treatment loss to follow-up, POC CD4+ testing has great potential to increase early ART initiation. However, additional diagnostic barriers, particularly the ancillary tests required to determine the appropriate first-line regimen after determination of ART eligibility, need to be removed in order to further increase linkage to treatment. Task-shifted POC CD4+ testing should be more widely implemented as an important tool to both increase access to treatment and improve the efficiency of ART services.

\section{Acknowledgements}

The findings and conclusions in this report are those of the authors and do not necessarily represent the official position 
of the Centers for Disease Control and Prevention or the Agency for Toxic Substances and Disease Registry.

\section{Competing interest}

We declare that we have no financial or personal relationships that may have inappropriately influenced us in writing this article.

\section{Sources of support}

This research was supported by the President's Emergency Plan for AIDS Relief (PEPFAR) through the Centers for Disease Control and Prevention under the terms of Award \#UPS001094A.

\section{Author contributions}

S.S., J.G. and G.M. conceived the study and drafted the manuscript. F.K.-T., N.S., M.N., C.M. and A.B. conducted the pilot and collected the data. A.D.M. performed the data analysis. A.M. and D.W.L. provided resources and oversaw the study.

\section{References}

1. UNAIDS. Report on the global AIDS epidemic [homepage on the Internet]. 2012 [cited 2015 Jun 15]. Available from: http://www.unaids.org/en/resources/ publications/2012/name,76121,en.asp

2. Lawn SD, Campbell L, Kaplan R, et al. Time to initiation of antiretroviral therapy among patients with HIV-associated tuberculosis in Cape Town, South Africa. Acquir Immune Defic Syndr. 2011;57(2):136-140. https://doi.org/10.1097/ QAl.0b013e3182199ee9

3. Karcher H, Omondi A, Odera J, Kunz A, Harms G. Risk factors for treatment denial and loss to follow-up in an antiretroviral treatment cohort in Kenya. Trop Med Int Health. 2007;12:687-694. https://doi.org/10.1111/j.1365-3156.2007.01830.x

4. Bassett IV, Wang B, Chetty S, et al. Loss to care and death before antiretroviral therapy in Durban, South Africa. J Acquir Immune Defic Syndr. 2009;51:135-139. https://doi.org/10.1097/QAl.0b013e3181a44ef2

5. Micek MA, Gimbel-Sherr K, Baptista AJ, et al. Loss to follow-up of adults in public HIV care systems in central Mozambique: Identifying obstacles to treatment. J Acquir Immune Defic Syndr. 2009;52:397-405. https://doi.org/10.1097/ QAI.0b013e3181ab73e2

6. Amuron B, Namara G, Birungi J, et al. Mortality and loss-to-follow-up during the pre-treatment period in an antiretroviral therapy programme under normal pre-treatment period in an antiretroviral therapy programme under normal
health service conditions in Uganda. BMC Public Health. 2009;9:290. https://doi. health service conditions in Ugand
org/10.1186/1471-2458-9-290

7. Losina E, Bassett IV, Giddy J, et al. The 'ART' of linkage: Pre-treatment loss to care after HIV diagnosis at two PEPFAR sites in Durban, South Africa. PLoS One. 2010;5:e9538. https://doi.org/10.1371/journal.pone.0009538

8. Rosen S, Fox M. Retention in HIV care between testing and treatment in subSaharan Africa: A systematic review. PLoS Med. 2011;8:e1001056. https://doi. org/10.1371/journal.pmed.1001056

9. Wynberg E, Cooke G, Shroufi A, et al. Impact of point-of-care CD4 testing on linkage to HIV care: A systematic review. J Int AIDS Soc. 2011;17:18809

10. Van Schaik N, Kranzer K, Mye L, et al. Field validation of the PIMA Analyzer in a mobile clinic setting in South Africa. Poster session presented at the 18th Conference on Retroviruses and Opportunistic Infections; 2011 Feb 27-Mar 2; Boston, MA.

11. Thakar M, Mahajan B, Shaikh N, et al. Utility of the point of care CD4 analyzer, PIMA, to enumerate CD4 counts in the field settings in India. AIDS Res Ther. 2012;9(1):26. https://doi.org/10.1186/1742-6405-9-26

12. Manabe YC, Wang Y, Elbireer A, Auerbach B, Castelnuovo B. Evaluation of portable point-of-care CD4 counter with high sensitivity for detecting patients eligible for
antiretroviral therapy. PLoS One. 2012;7(4):e34319. https://doi.org/10.1371/ journal.pone.0034319

13. Glencross DK, Coetzee LM, Faal M, et al. Performance evaluation of the Pima ${ }^{T M}$ point-of-care CD4 analyzer using capillary blood sampling in field tests in South Africa. J Int AIDS Soc. 2012;15:3. https://doi.org/10.1186/1758-2652-15-3

14. World Health Organization Prequalification of Diagnostics Programme. Public report, product: Pima CD4 test. Number: PQDx 0099-032-00, Dec. 2011, version 2.0. Geneva: WHO; 2011.
15. Jani IV, Sitoe NE, Alfai ER, et al. Effect of point-of-care CD4 cell count tests on retention of patients and rates of antiretroviral therapy initiation in primary health clinics: An observational cohort study. Lancet. 2011;378(9802):1572-1579. https://doi.org/10.1016/S0140-6736(11)61052-0

16. Faal M, Naidoo N, Glencross DK, et al. Providing immediate CD4 count results at HIV testing improves ART initiation. J Acquir Immune Defic Syndr. 2011;58:e54-e59. https://doi.org/10.1097/QAI.1090b1013e3182303921

17. Larson BA, Bistline $K$, Ndibongo B, et al. Rapid point-of-care CD4 testing at mobile HIV testing sites to increase linkage to care: An evaluation of a pilot program in South Africa. Oral abstract presented at the 6th IAS Conference on HIV Pathogenesis and Treatment; 2011 July 17-20; Rome, Italy. Abstract MOAD0103.

18. Van Rooyen $\mathrm{H}$, Barnabas $\mathrm{R}$, Phakathi Z, et al. High testing uptake and linkages to HIV treatment through home-based HIV counseling and testing and facilitated referral: KwaZulu-Natal, South Africa. Oral abstract presented at 19th Conference on Retroviruses and Opportunistic Infections; 2012 March 5-8; Seattle, WA. Abstract 1135 .

19. World Health Organization. Task shifting: Global recommendations and guidelines [homepage on the Internet]. 2012. [cited 2015 Jun 15]. Available from: http://www.who.int/workforcealliance/knowledge/resources/taskshifting globalrecommendations/en/

20. Babigumira JB, Castelnuovo B, Lamorde $M$, et al. Potential impact of task-shifting on costs of antiretroviral therapy and physician supply in Uganda. BMC Health Serv Res. 2009;9:192. https://doi.org/10.1186/1472-6963-9-192

21. Callaghan M, Ford N, Schneider H. A systematic review of task- shifting for HIV treatment and care in Africa. Hum Resour Health. 2010;8:8. https://doi.org/ 10.1186/1478-4491-8-8

22. Bemelmans $\mathrm{M}$, Van den Akker $\mathrm{T}$, Ford $\mathrm{N}$, et al. Providing universal access to antiretroviral therapy in Thyolo, Malawi through task shifting and decentralization
of HIV/AIDS care. Trop Med Int Health. 2010;15:1413-1420. https://doi.org/ of HIV/AIDS care. Trop Med Int Heat
$10.1111 / \mathrm{j} .1365-3156.2010 .02649 . x$

23. Wet $\mathrm{K}$, Wouters $\mathrm{E}$, Engelbrecht $\mathrm{M}$. Exploring task-shifting practices in antiretroviral treatment facilities in the Free State Province, South Africa. J Public Health Policy. 2011;32(Suppl 1):S94-101. https://doi.org/10.1057/jphp.2011.30

24. Vers LC, Jerome JG, Cullen KA, et al. Task-shifting in HIV care: A case study of nurse-centered community-based care in Rural Haiti. PLoS One. 2011;6:e19276. https://doi.org/10.1371/journal.pone.0019276

25. Morris $\mathrm{MB}$, Chapula $\mathrm{BT}$, Chi BH, et al. Use of task-shifting to rapidly scale-up HIV treatment services: Experiences from Lusaka, Zambia. BMC Health Serv Res. 2009;9:5. https://doi.org/10.1186/1472-6963-9-5

26. Shumbusho F, Van Griensven J, Lowrance D, et al. Task shifting for scale-up of HIV care: Evaluation of nurse-centered antiretroviral treatment at rural health centers in Rwanda. PLoS Med. 2009;6:e1000163. https://doi.org/10.1371/journal.pmed. 1000163

27. Shetty AK, Mhazo M, Moyo S, et al. The feasibility of voluntary counselling and HIV testing for pregnant women using community volunteers in Zimbabwe. Int J STD AIDS. 2005;16:755-759. https://doi.org/10.1258/095646205774763090

28. Ledikwe JH, Kejelepula M, Maupo K, et al. Evaluation of a well-established taskshifting initiative: The lay counselor cadre in Botswana. PLoS One. 2013;8(4):e61601. https://doi.org/10.1371/journal.pone.0061601

29. Sanjana P, Torpey K, Schwarzwalder A, et al. Task-shifting HIV counseling and testing services in Zambia: The role of lay counsellors. Hum Resour Health. 2009;7:44. https://doi.org/10.1186/1478-4491-7-44

30. Namibia national guidelines for antiretroviral therapy. 3rd ed. [homepage on the Internet]. 2010. [cited 2017 Jun 30]. Available from: http://apps.who.int/ medicinedocs/en/d/Js21939en

31. Namibia national guidelines for antiretroviral therapy. 4th ed. [homepage on the Internet]. 2014. [cited 2017 Jun 30]. Available from: https://www.medbox.org/ namibia/namibia-national-guidelines-for-antiretroviral-therapy-fourth-edition/ preview

32. World Health Organization. Consolidated guidelines on the use of antiretrovira drugs for treating and preventing HIV infection [homepage on the Internet]. 2013. [cited 2015 Jun 15]. Available from: http://www.who.int/hiv/pub/guidelines/ arv2013/download/en/

33. Fernandez-Fernandez B, Montoya-Ferrer A, Sanz AB, et al. Tenofovir nephrotoxicity: 2011 update. AIDS Res Treat. 2011;2011:354908. https://doi.org/ 10.1155/2011/354908

34. Young J, Schäfer J, Fux CA, et al. Renal function in patients with HIV starting therapy with tenofovir and either efavirenz, lopinavir or atazanavir. AIDS. 2012;26:567-575. https://doi.org/10.1097/QAD.0b013e32834f337c

35. World Health Organization. Hepatitis B surface antigen assays: Operational characteristics (Phase I), Report 1, blood safety and clinical technology [homepage on the Internet]. 2001. [cited 2015 Jun 15]. Available from: http://www.who.int/ diagnostics_laboratory/studiestudys/en/hep_B_rep1.pdf

36. Cholestech Corporation. The accuracy and reproducibility of a rapid, Fingerstick method for measuring alanine aminotransferase is comparable to laboratory reference methods. Technical Brief ML1255-01A. Hayward, CA: Cholestech; 2001.

37. Pollock NR, Rolland JP, Kumar S, et al. A paper-based multiplexed transaminase test for low-cost, point-of-care liver function testing. Sci Transl Med. 2012;4(152):152ra129. https://doi.org/10.1126/scitranslmed.3003981

38. Centre for Evidence-based Purchasing. Point of care tests for the measurement of creatinine. CEP10042. Study report [homepage on the Internet]. 2010. [cited 2015 Jun 15]. Available from: http://nhscep.useconnect.co.uk/CEPProducts/ Catalogue.aspx 